\title{
Development of New Collaborative Design and Engineering Environment
}

\author{
A.Ohtaka and S.Sasao \\ Nihon Unisys, Ltd. \\ 1-1-1 Toyosu, Koto-ku, Tokyo 135-8560, Japan \\ Telephone: $+81-3-5546-4784$ \\ Facsimile : $+81-3-5546-7810$ \\ E-mail : akihiko.ohtaka@unisys.co.jp \\ shinobu.sasao@unisys.co.jp
}

\begin{abstract}
The present day advanced mechanical CAD systems support wide variety of sophisticated capabilities. But, they are essentially aimed at single engineer's use. Drastic productivity improvement requires an evolution of CAD/CAM systems so that they are appropriate for two or more engineers simultaneous use.

The present paper discusses a collaboration support environment realized in CADCEUS, which is an integrated CAD/CAM/CAE/CG system developed by Nihon Unisys. Major technical focuses for its development has been adoption of SWS(Shared Work Space) and PWS(Private Work Space) concepts in DBMS and effective use of parametric modeling capability for the automatic consistency management of design data.

For confirming the effectiveness of the new collaboration support environment, it is applied to actual automobile engine design from early fundamental design through detailed design which requires collaboration and automatic consistency management of constituent models in various levels.
\end{abstract}

\section{Keywords}

Collaborative design, parametric modeling, assembly modeling, shared work space, update right, simultaneous use 


\section{INTRODUCTION}

Authors have established a framework and some application functions on top of CADCEUS ${ }^{* 1}$ for realizing collaborative design support. Capabilities developed enable automatic consistency management of two or more simultaneously working engineers activities and related design data, which is required in the collaborative design environment such as the design of automobile engine. Technical focus for realizing these capabilities was the combination of database management capability with hybrid modeling capability.

This paper first makes clear requirements of CAD/CAM system framework for realizing collaborative design support. Then, newly developed collaboration support functions where SWS ( Shared Work Space) plays a central role is described in detail.

Chapter-2 discusses background where collaborative design is necessitated, and requirements in application level. Chapter-3 defines requirements of framework for supporting collaborative design, and Chapter-4 discusses newly developed framework and capabilities which satisfy defined requirements. Chapter-5 shows practical application example, and Chapter- 6 summarizes future extensions.

\section{BACKGROUND OF COLLABORATIVE DESIGN AND REQUIREMENTS}

In manufacturing industry, reduction of lead time through whole manufacturing processes and further improvement of product quality are still very important issues. For resolving these issues, thorough quality management by TQC(Total Quality Control)has been practiced, and concurrency of manufacturing processes has been pursued.

As for the reduction of time required in each manufacturing process, major focus has been sophistication of CAD/CAM capabilities, and promotion of automation. It can be said that various challenges for reducing time necessary for product development has been tried on the condition that ' $\mathrm{CAD} / \mathrm{CAM}$ system is a tool for single engineer's use'.

But, in view of the fact that two or more engineers are simultaneously concerned for the design of almost all large scale design object, we can not essentially resolve the issue unless we establish a framework which directly support consistency management of two or more engineers simultaneous activities. In other words, evolution which makes CAD/CAM system 'a common tool for two or more engineers simultaneous use' in place of the conventional situation that CAD/CAM system is 'a tool for single engineer's use' is necessary.

\footnotetext{
${ }^{*}:$ CADCEUS is the integrated CAD/CAM/CAE/CG system developed by Nihon Unisys. CADCEUS is adopted as the baseline system of Toyota's 'TOGO' system, and has many users especially in automobile industry.
} 
We frequently hear the claim, as one of demerits of introducing CAD system, that it became more difficult to confirm consistency of simultaneously working engineers activities, to judge whole product quality, and to judge progress of design than conventional explicit drafting based design mainly because of each engineer's design quality and progress are hidden in a CAD terminal or in his personal database. The major reason which give rise to this claim will be lack of evolution for making CAD system a common tool for two or more engineers simultaneous use.

We then consider how to categorize design activities for extracting requirements of collaboration support. The author categorizes them into two types. First type is requirements of activity management level, and the second is requirements while actual design work is proceeded. Major requirements in these types are as follows. Requirements of activity management level

(1) Capability for supporting the judgment of a project manager such as a capability for grasping whole design status, and that for transmitting his instruction to engineers should be provided.

(2) Capability for transmitting and maintaining fundamental shape and constraints determined in fundamental design phase to detailed design phase(engineers) should be provided.

(3) Common information and personal information should be clearly distinguished, and common information should be freely referenced.

(4) Inconsistency between engineers should be entirely detected, announced user friendly, and hopefully adjusted automatically.

Requirements while design is proceeded

(1) Capability for showing an engineer the latest status of other engineers work which may affect him should be provided.

(2) Capability to transfer update right is necessary. This capability is required in the situation that responsible area for each engineer had better be changed according to the progress of detailed design.

(3) Capability for announcing an engineer the change of other engineer's work which he is referencing is necessary. Revision of his model corresponding to the latest status of other engineer's work had better be performed with minimum operation.

(4) Each engineer's work should be independently performed with maximum efficiency without interrupts.

(5) Free communication between related engineers by the use of various media had better be supported.

\section{REQUIREMENTS OF CAD/CAM SYSTEM FRAMEWORK}

In order to satisfy requirements described in the previous chapter, framework of $\mathrm{CAD} / \mathrm{CAM}$ system is required to have cooperative and distributed database management capability, namely a mechanism to provide both personal design space and common design space, and to maintain consistency of these two spaces 
is required. Capabilities necessary in conjunction with this cooperative and distributed database management are;

(1) Realization of product model

(2) Realization of parametric modeling capability including assembly parametrics

(3) Provision of flexible communication means between engineers

\subsection{Realization of cooperative and distributed database management (DBMS)}

Fundamental requirements of collaborative design is that related engineers can share a model. Namely, DBMS which enables engineers to share logically single data model is required irrespective of the representation of the data model.

For realizing this requirement, DBMS is necessary to provide the following capabilities;

(1) exclusive control

(2) possession of private data( half boiled data, data which should be hidden from other engineers, etc.)

(3) notice of the influence of some engineer's design change

Each engineer perform independent work for some design period. In that period, data which is not necessary to be shared by other engineers such as data which is under investigation (half boiled data) exist. Mechanism to store these data temporarily is also necessary other than data sharing mechanism. These studies reveals that cooperative and distributed DBMS is indispensable.

Notice of one engineers operation on shared data to other engineers is also an important requirement for realizing collaborative design support.

\subsection{Realization of product model}

Shared information among engineers includes not only 3D shape model, but also non-geometric information such as design parameters, design intention, design process, and criteria at each design process. Therefore, it becomes indispensable for supporting collaborative design to realize product model where these nongeometry information is adequately related to shape model.

\subsection{Realization of high level shape modeling engine}

It is crucially important to provide a modeling engine which enables flexible representation including non-manifold representation, deformation of design object, and re-use of existing models.

Here, requirement on representation means representation of design object with arbitrary mixture of solid, surface and wireframe models. It naturally requires nonmanifold representation. The reason the author thinks so is that above mixture is the best model, at the moment, judging from the cost required in solid modeling. 
Requirement on shape deformation is to provide parametric capability for a part model represented by above described mixture model, and for an assembly model represented by combining those part models.

As for the re-usability, specific explanation will not be necessary. When we categorize manufacturing activities into two types ; real new product development and major or minor modification of existing models, the ratio of the latter is overwhelming. Central technology for supporting re-usability is also a parametric technology.

\subsection{Provision of communication means}

In addition to the mechanism to share data, communication which supports effective simultaneous activities is also important for realizing collaboration support. Obvious requirements are;

(1) Assembly viewing capability which some commercial systems support is one of good means for a project manager to use for grasping whole design status.

(2) Free hand instruction writing on the manager's terminal and its transmission to indicated engineer greatly improves communication between project manager and engineers.

(3) Remote conference capability which enable virtual meeting among engineers and share same data on their terminals is effective when design is simultaneously performed by engineers located in far distant places.

(4) Use of CG data is also effective for referencing past data.

See Sekido(1997) for (2) and (3).

Recent drastic advancement of network capability has strong possibility to improve communication speed and contents.

\section{REALIZED COLLABORATION SUPPORT FRAMEWORK AND CAPABILITIES}

This chapter describes collaboration support environment realized from two points, namely database management capability and modeling capability.

\subsection{Database management capability}

Before going detailed explanation of developed capabilities, overview of CADCEUS data model is as follows. Hierarchy of data set is;

Work Space(WS) - Object(OBJ) - Group(GRP) - Entity(ENT)

Here, ENTITY is a minimum and leaf constituent of a product model, such as geometric entity, topological entity. Group is a concept to arbitrary summarize them. Object is a concept corresponding to a part, or an assembly, or a drawing sheet. Objects can have arbitrary hierarchy. Work Space is the highest concept among data set for representing a product model, and it corresponds to a design space. One Work Space can include any number of Objects. Work Space and Group are not allowed to have hierarchy. 
(1) Realization of Shared Work Space(SWS)

Fundamental requirement of collaboration support again is ' to realize shared design space of related engineers satisfying maximum independence and efficiency of each engineer's work'.

In order to realize this requirement, we separated WS into two types, one is PWS(Private Work Space) for personal use , the other is SWS(Shared Work Space) for common use, and developed a SIM(Shared Information Manager) which manages transfer of data between SWS and PWS. Those information stored in SWS are fundamental design information(shape and constraints of the shape) common to all engineers, information which span two or more engineers, and objects which is disclosed to all engineers. Each engineer imports fundamental design information and other engineer's OBJ which is related to his design work from SWS. Then, for the time being, he proceeds his design work independently. SIM always monitors information on SWS, and from what engineer the information on SWS is referenced. When some OBJ referenced by other engineer is updated on SWS, then SIM informs referencing engineer the fact and urges him to re-import the latest $\mathrm{OBJ}$. When all the engineers finish their respective design and register their OBJs to SWS, then whole design is completed. The relation among SWS, PWS and SIM is as shown in Figure-1.

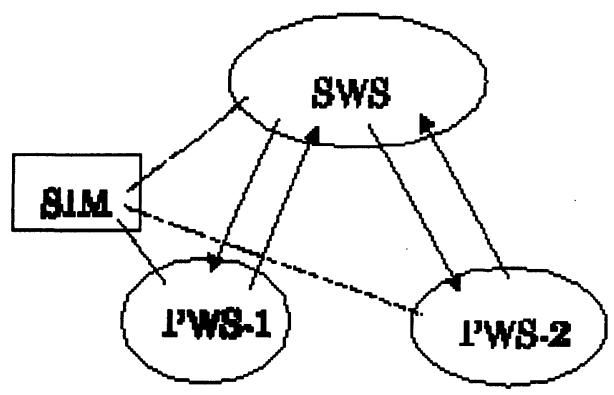

Figure 1: Fundamental structure of collaboration support.

In this mechanism, SWS is visible from all engineers but PWS is invisible from other engineers, which satisfy a requirement concerning independence of each engineer's work.

When the change of the other engineer's OBJ referencing occurs, concerning message is passed from SIM. What he should do ,at first, is to receive the latest status of the other engineer's OBJ from SWS. Then, he can automatically reconstruct his $\mathrm{OBJ}$ consistent with the newly received $\mathrm{OBJ}$ by the use of parametric capability described later.

As for the project manager's activity support of checking each engineer's design quality and progress, it is necessary to regulate engineers to register their under 
design OBJs to SWS with pre-defined cycle. Then, the project manager can check whole design status, each engineer's design quality and progress on SWS. Message passing capability of SIM can be used for sending the project manager's instruction to each engineer.

(2) Update right control of $\mathrm{OBJ}$

OBJs on SWS can be assigned update right by the use of SIM. Update right of one $\mathrm{OBJ}$ is assigned to only one engineer. One engineer may have two or more OBJs of which update right he has. Those OBJs of which update right are possessed by other engineer can be used and modified on PWS but can not be transferred to SWS.

(3) Consistency management of data model between engineers

'OBJ reflection capability' is prepared for revising referencing other engineer's $\mathrm{OBJ}$ to the latest status. This is a capability to transfer requested OBJ from SWS to requester specified work space via SIM. If the OBJ to be revised has any relation with other OBJs such as placement relation or reference dependency relation, SIM automatically recognize the situation and urges the engineer to execute parametric re-construction. History based parametric re-construction capability enable the engineer to obtain his re-constructed $\mathrm{OBJ}$ consistent with the latest status of other engineer's OBJs.

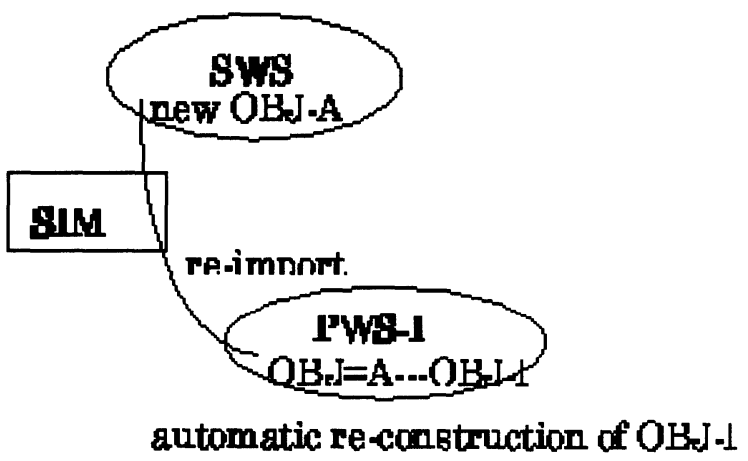

Figure 2: Mechanism of consistency management among engineers.

(4) Common information

Every time OBJ is registered to SWS, a message called 'common information' is passed to all other engineers. This message inform what engineer has done what to what OBJ. Each engineer can judge its influence to his OBJ. If he judges the necessity of reflecting the change to his model, then he can do it by the use of previously described $\mathrm{OBJ}$ reflection capability.

(5) Check out reservoir

Usually, design period by the use of CAD/CAM system is considerably long. It means that it is required to keep under design data for a long time. Since SWS is a 
public space, engineers do not register their models unless they understand that design is almost completed and their model could be referenced by other engineers. In order to keep under design data, the system provides check out reservoir corresponding to each PWS. By the use of this mechanism, an engineer can keep his under investigation data for necessary period without interruption by other engineers.

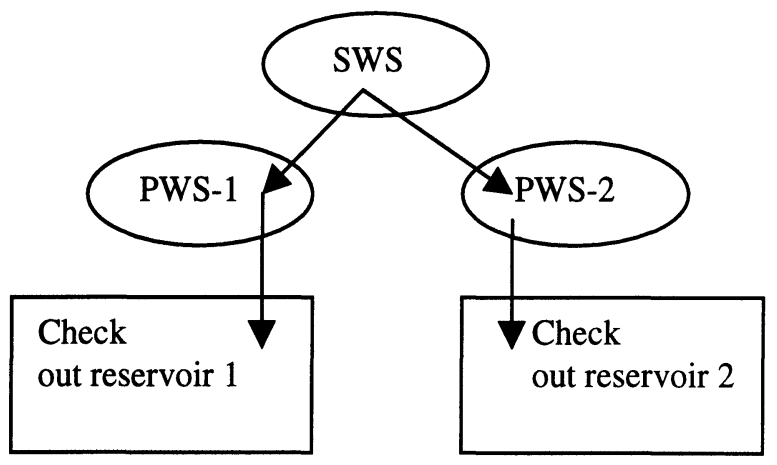

Figure 3: Check out reservoir.

(6) Global parameter

Product model is already implemented in CADCEUS such that product configuration data, assembly model, FEM data, kinematics data, NC related data are stored in database with necessary relations with product shape data.

This section describes 'global parameter' which has been newly introduced for supporting collaborative design. Global parameter is user definable parameters mainly for sharing design parameters among engineers. By possessing various parameters defined in the fundamental design phase as global parameters, further detailed design could be simultaneously performed sharing design parameters. Furthermore, by changing some global parameters and by automatically reconstructing related parts, influence of parameter change can be easily reflected on product shape. Global parameters are stored on SWS and therefore visible from all the engineers. Each engineer at first import global parameters onto his PWS, and performs his design consistent with global parameters defined in fundamental design phase by relating dimensions of his model with global parameters by the use of algebraic constraint definition capability. Global parameters may be changed on his PWS independently for investigating various design plan though the change can not be exported to SWS. These situations are illustrated in Figure-4. See Sasao(1998) for the details of global parameters. 


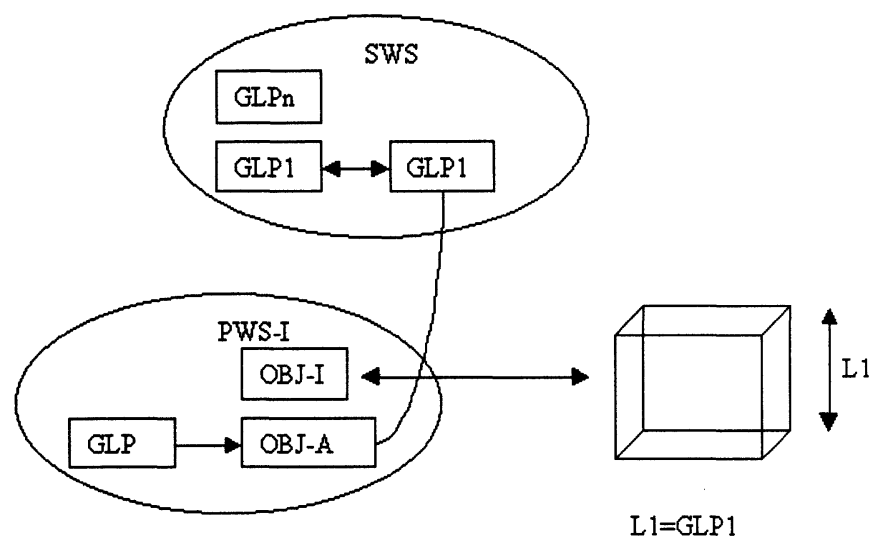

Figure 4: Global parameter.

\subsection{Hybrid modeling}

CADCEUS realized flexible and powerful modeling which is a hybrid modeler in every sense. Fundamental recognition for realizing it was the following two points ;

1) Not every design models are suited for solid modeling.

2) Not every design models are suited for feature based modeling.

Based on this recognition, we have developed a hybrid modeler which has the following characteristics.

1) The user can represent his model with arbitrary mixture of solid, surface, and wireframe models.

2) The user can represent his model with mixture of feature based representation and conventional(non-feature based) representation.

3) Parametric modeling capability works not only for solid model, but also for surface model, wireframe model, and their any mixture.

Though CADCEUS parametric modeling mechanism itself is the combination of geometric constraints, positional constraints and history based model as is implemented in some other advanced CAD systems, extension of object model to surface model and wireframe model drastically enhanced applicability to practical design problems. Since the system memorizes reference dependency relation during modeling, and has a capability to re-construct a model(part model or assembly model) after the change of referenced entities, automatic reflection of other engineers' OBJ change is possible.

\section{EXAMPLE}

This chapter examines the effectiveness of collaboration support capability developed by using automobile engine design as an example. Supposed story is as follows; 
1) There is only one fundamental designer. After he finished fundamental design, he registers fundamental shape(skelton) and global parameters to SWS as shown in Figure-5.

2) There are three detailed designers, one for exhaust port design, another for water jacket design, and the other for cylinder head design.

3) After receiving fundamental shape and global parameters, port designer proceed his design. When almost all shape is determined, he checks the design quality of his model by analysing distribution of sectional area, and finds out low quality portion where sectional area acutely changes as shown in Figure-6.

He then corrects base curve of the port and automatically re-construct his model by the use of parametric capability as shown in Figure-7. The reader may understand the improvement of sectional area distribution. Finally, he register his completed model to SWS.

4) Water jacket designer, when announced the revision of referencing port data, re-import new port data from SWS and re-construct his model corresponding to the port data in the same way. Cylinder head designer may also re-construct his model in the same way after importing latest port and water jacket.

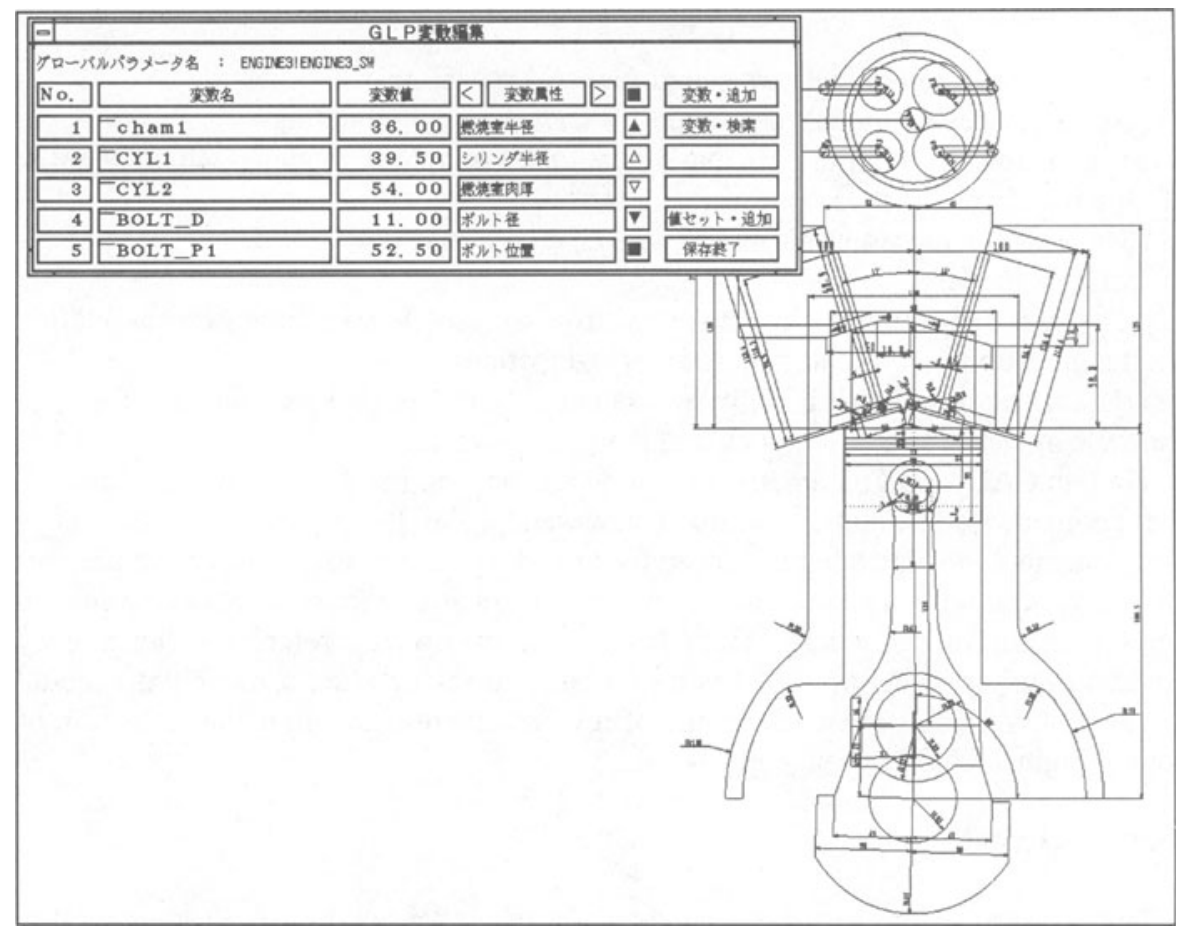

Figure 5: Fundamental design data and global parameters. 


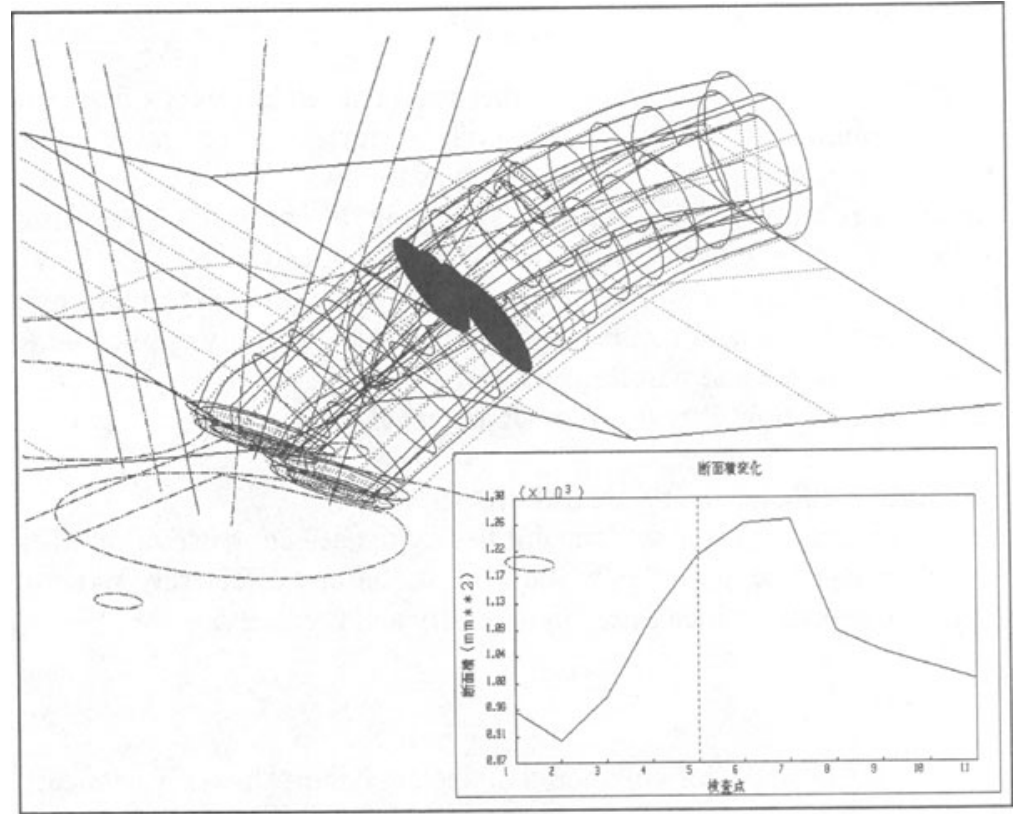

Figure 6: Quality analysis of a port.

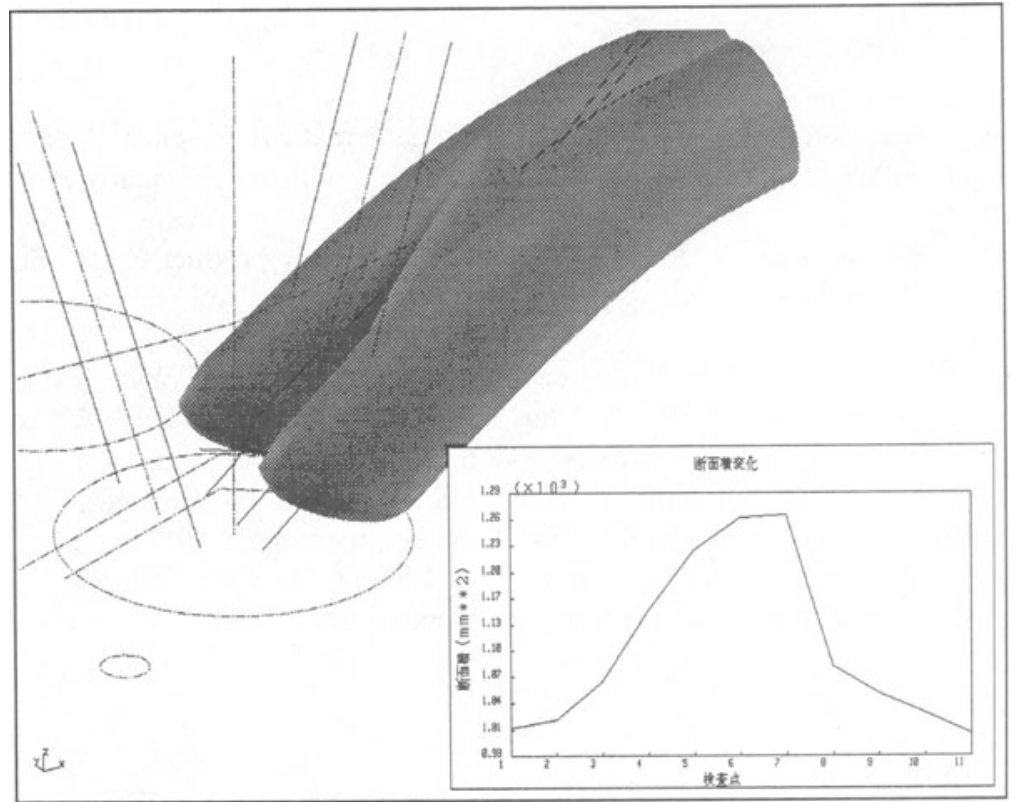

Figure 7: Re-constructed port. 


\section{CONCLUSION}

A new collaborative design support environment which is a first step for realizingan evolution towards 'CAD system for two or more engineers simultaneous use' is presented. The results are as follows;

(1) Common design space is realized satisfying a constraint that maximum independence of each engineer's work should be guaranteed.

(2) Automatic re-construction of design model, when referencing other engineer's model is changed, is realized by the use of parametric capability applicable for any mixture of solid, surface and wireframe models.

(3) Control of update right is realized in Object level.

Issues for further enhancements are as follows.

(1) Extend communication support capabilities by the effective use of multi-media.

(2) Improve manager support capabilities so that he can effectively judge design quality and progress of each engineer more easily and precisely.

\section{REFERENCES}

Sasao,s.(1998) CADCEUS for collaborative design, Nihon Unisys Technical Report(to be published)

Sekido,t.(1997) Development of collaborative design support capability, Nihon

Unisys Technical Symposium Proceedings(to be published)

\section{BIOGRAPHY}

Shinobu Sasao received B.S. degree of mathematical science from Keio University, Japan in 1981. He has been concerned with mechanical CAD/CAM systems

development since then. As for Nihon Unisys proprietary product CADCEUS, he has developed database module as the module leader.

Akihiko Ohtaka received M.S. degree of mathematics from Tokyo Institute of Technology, Japan in 1970. He has been concerned with FEM system developmentand mechanical CAD/CAM systems development since then.

$\mathrm{He}$ has initiated the development of CADCEUS as the principal designer.

$\mathrm{He}$ has also been concerned with ISO/STEP development these 10 years.

He has contributed to the development of Part42:Shape Representation,

Part105:Kinematics,and now developing parametrics standard. 